\title{
Adaptive optics near infrared integral field spectroscopy of NGC 2992 ${ }^{\star}$
}

\author{
S. Friedrich ${ }^{1}$, R. I. Davies ${ }^{1}$, E. K. S. Hicks ${ }^{1}$, H. Engel ${ }^{1}$, F. Müller-Sánchez ${ }^{1,2}$, R. Genzel ${ }^{1,3}$, and L. J. Tacconi ${ }^{1}$ \\ 1 Max-Planck-Institut für extraterrestrische Physik, Postfach 1312, 85741 Garching, Germany \\ e-mail: sfriedrich@mpe.mpg.de \\ 2 Instituto de Astrofísica de Canarias, 38205 La Laguna (Tenerife), Spain \\ 3 Department of Physics, Le Conte Hall, University of California, Berkeley, CA 94720, USA
}

Received 21 December 2009 / Accepted 12 May 2010

\section{ABSTRACT}

\begin{abstract}
Aims. NGC 2992 is an intermediate Seyfert 1 galaxy showing outflows on kilo parsec scales which might be due either to AGN or starburst activity. We therefore aim at investigating its central region for a putative starburst in the past and its connection to the AGN and the outflows.

Methods. Observations were performed with the adaptive optics near infrared integral field spectrograph SINFONI on the VLT, complemented by longslit observations with ISAAC on the VLT, as well as $N$ - and $Q$-band data from the Spitzer archive. The spatial and spectral resolutions of the SINFONI data are $50 \mathrm{pc}$ and $83 \mathrm{~km} \mathrm{~s}^{-1}$, respectively. The field of view of $3^{\prime \prime} \times 3^{\prime \prime}$ corresponds to $450 \mathrm{pc} \times 450 \mathrm{pc}$. Br $\gamma$ equivalent width and line fluxes from PAHs were compared to stellar population models to constrain the age of the putative recent star formation. A simple geometric model of two mutually inclined disks and an additional cone to describe an outflow was developed to explain the observed complex velocity field in $\mathrm{H}_{2} 1-0 \mathrm{~S}(1)$.

Results. The morphologies of the $\operatorname{Br} \gamma$ and the stellar continuum are different suggesting that at least part of the Br $\gamma$ emission comes from the AGN. This is confirmed by PAH emission lines at $6.2 \mu \mathrm{m}$ and $11.2 \mu \mathrm{m}$ and the strength of the silicon absorption feature at $9.7 \mu \mathrm{m}$, which point to dominant AGN activity with a relatively minor starburst contribution. We find a starburst age of 40-50 Myr from $\mathrm{Br} \gamma$ line diagnostics and the radio continuum; ongoing star formation can be excluded. Both the energetics and the timescales indicate that the outflows are driven by the AGN rather than the starburst. The complex velocity field observed in $\mathrm{H}_{2} 1-0 \mathrm{~S}(1)$ in the central $450 \mathrm{pc}$ can be explained by the superposition of the galaxy rotation and an outflow.
\end{abstract}

Key words. galaxies: active - galaxies: individual: NGC 2992 - galaxies: Seyfert - galaxies: starburst - infrared: galaxies

\section{Introduction}

NGC 2992, initially classified as a Seyfert 2, was later classified as an intermediate Seyfert 1 galaxy on the basis of a broad $\mathrm{H} \alpha$ component with no corresponding $\mathrm{H} \beta$ component in its nuclear spectrum (Ward et al. 1980). Later, Glass (1997) suggested that it is a hybrid between an intermediate Seyfert and a starburst galaxy, induced by the interaction with NGC 2993 to the south. Both galaxies are connected by a tidal tail with a projected length of 2 ! 9 .

NGC 2992 is highly inclined by about $70^{\circ}$ to our line of sight with a broad disturbed lane of dust in its equatorial plane. The redshift is 0.0077 which corresponds to a distance of $32.5 \mathrm{Mpc}$ and an angular scale of $150 \mathrm{pc}$ per arcsec assuming $H_{0}=75 \mathrm{~km} \mathrm{~s}^{-1} \mathrm{Mpc}^{-1}$.

At a wavelength of $6 \mathrm{~cm}$ NGC 2992 shows prominent loops to the northwest and southeast. This feature, designated as figure-of- 8 , has a size of about $8^{\prime \prime}$ with the long axis at a position angle (PA) of $-26^{\circ}$, which is not coincident or perpendicular to the major axis (Ulvestad \& Wilson 1984). Chapman et al. (2000) conclude from their IR observations that the most convincing interpretation for these loops is that of expanding gas bubbles which are seen as limb-brigthened loops as was already proposed by Wehrle \& Morris (1988). Two possible explanations

* Based on observations collected at the European Southern Observatory, Chile (074.B-9012). are discussed for the origin of the loops: they may be driven by an AGN or a superwind from a compact starburst. X-ray observations point to the first possibility: Colbert et al. (1998) concluded that the large scale outflows are driven by nonthermal jets from the AGN that entrain the surrounding material and heat it by shocks over kpc spatial scales.

Heckman et al. (1981) reported complex kinematics in NGC 2992 on the basis of longslit spectra at PA $=120^{\circ}$. This work was extended by Colina et al. (1987) who find indications for an outflow within a plane highly inclined to the plane of the galaxy. Márquez et al. (1998) measured [O III] and $\mathrm{H} \alpha$ lines at nine different position angles and concluded, although from a simple kinematical model, that the double peaked line profiles and asymmetries can be accounted for by an outflow within a conical envelope or on the surface of a hollow cone. The high

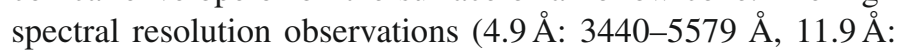
$5840-9725 \AA$ ) of narrow line regions in NGC 2992 of Allen et al. (1999) show at most positions a double component line profile. One component of the double peaked lines follows the galactic rotation curve, while the other is identified as a wind that is expanding out of the plane of the galaxy with a velocity of up to $200 \mathrm{~km} \mathrm{~s}^{-1}$. These authors did not find spectral evidence for a significant contribution from a starburst to the outflow.

Depending on the inclinations of galaxy and cone several interpretations of these kinematics are possible. It is generally assumed that the NW edge of the disk of NGC 2992 is closest 
to us with trailing spiral arms, and that the SE cone is closer and directed at us. However, an arc-shaped emission found by García-Lorenzo et al. (2001), which might be associated with the radio loop, is red shifted in the SE, and can therefore only be consistent with an outflow if it is pointing away from us.

In this paper, we present spatially and spectrally resolved $K$-band observations of the inner $3^{\prime \prime} \times 3^{\prime \prime}$ of NGC 2992 taken with the adaptive optics integral field spectrograph SINFONI. These are complemented by longslit observations along the major axis out to a radius of about $5^{\prime \prime}$ with ISAAC, and $N$ - and $Q$-band spectra from the Spitzer archive. SINFONI data were taken with unprecedent spatial resolution in the $K$-band which allows us to study the dynamics of gas and stars in the central region in detail. Together with the spectral resolution of SINFONI this enables us to search for a starburst and its connection to the AGN and, possibly, to the outflows. After presenting the observational results in Sect. 3, we discuss the observations in Sect. 4, and show that there is evidence for a nuclear starburst in NGC 2992. Finally, in Sect. 5 we concentrate on the dynamics of the gas and stars and the consequences on the geometry of the galaxy.

\section{Observations and data reduction}

\subsection{SINFONI}

$K$-band spectra of NGC 2992 between approximately $1.95 \mu \mathrm{m}$ and $2.45 \mu \mathrm{m}$ were taken on 13 March 2005 at the VLT with SINFONI, an adaptive optics near infrared integral field spectrograph (Eisenhauer et al. 2003; Bonnet et al. 2004) with a spectral resolution of 3600 . The pixel scale was $0 .{ }^{\prime} 05 \times 0.0^{\prime} 1$ and the exposure time $600 \mathrm{~s}$ for both object and sky frames. Object frames were interspersed with sky frames using the sequence O-S-O-O-S-O. A total of 12 object frames were obtained, resulting in a total exposure time of two hours. For a near diffraction limited correction the nucleus of the galaxy was used by the AO module.

Data were reduced in a standard manner by subtraction sky frames, flatfielding, correcting bad pixels, and wavelength calibration with an arc lamp using the SPRED software package (Abuter et al. 2006), which also performs the reconstruction of the data cube. No spatial smoothing by a median filter was applied. Telluric correction and flux calibration were performed with the G2V star HD 85724 ( $K=7881)$. Residuals from the $\mathrm{OH}$ line emission were removed using the method described in Davies (2007a).

The spatial resolution has been estimated from both the broad Br $\gamma$ and the non-stellar continuum (Davies 2007b). Both methods yield symmetric PSFs with FWHMs of 0.'32 and 0.'29, respectively, corresponding to $48 \mathrm{pc}$ and $44 \mathrm{pc}$, respectively. The spectral resolution derived from sky lines is $83 \mathrm{~km} \mathrm{~s}^{-1}$. NGC 2992 is part of a (heterogeneous) sample of type 1 and type 2 Seyfert galaxies, ULIRGs, and a Quasar, which were observed to investigate the connection between star formation and AGN. Further details on data reduction can be found in Davies et al. (2007) and Hicks et al. (2009).

\subsection{ISAAC}

NGC 2992 was also observed on 13 January 2006 with the Infrared Spectrometer And Array Camera ISAAC at the VLT in order to obtain wide FOV longslit spectra centered on $2.1 \mu \mathrm{m}$ (to measure $\mathrm{H}_{2} 1-0 \mathrm{~S}(1)$ ) and $2.29 \mu \mathrm{m}$ (to measure ${ }^{12} \mathrm{CO}(2-0)$ ). The $2^{\prime}$ long and 0.3 wide slit was aligned parallel to the

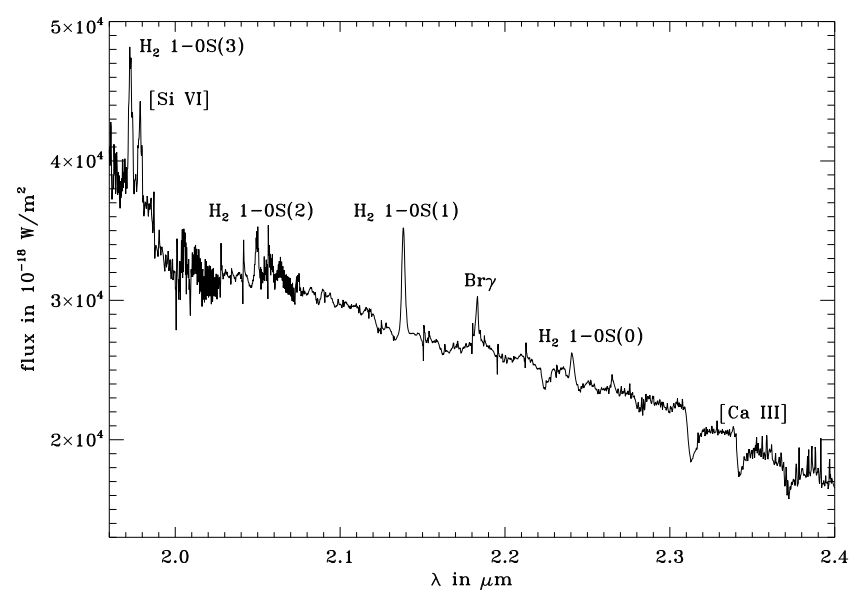

Fig. 1. SINFONI-Spectrum of NGC 2992 integrated over the whole FOV of $3^{\prime \prime} \times 3^{\prime \prime}$. The most prominent lines are those of $\mathrm{H}_{2} 1-0 \mathrm{~S}(3)$ at $1.958 \mu \mathrm{m},[\mathrm{Si} \mathrm{VI}]$ at $1.96 \mu \mathrm{m}, \mathrm{H}_{2} 1-0 \mathrm{~S}(1)$ at $2.12 \mu \mathrm{m}$ and $\mathrm{Br} \gamma$ at $2.16 \mu \mathrm{m}$.

Table 1. Measured line fluxes for NGC 2992 in a field of view of 1".5 $\times$ 1 ." 5 centered on the nucleus.

\begin{tabular}{lrrr}
\hline \hline Line & $\begin{array}{c}\lambda^{a} \\
(\mu \mathrm{m})\end{array}$ & $\begin{array}{c}\text { Flux } \\
\left(10^{-18} \mathrm{~W} \mathrm{~m}^{-2}\right)\end{array}$ & $\begin{array}{l}\text { Flux }^{c} \\
\left(10^{-18} \mathrm{~W} \mathrm{~m}^{-2}\right)\end{array}$ \\
\hline $\mathrm{H}_{2} 1-0 \mathrm{~S}(3)$ & 1.9576 & $11 \pm 0.2$ & 5 \\
{$[$ Si VI] } & 1.9634 & $13 \pm 1.6$ & 4 \\
$\mathrm{H}_{2} 1-0 \mathrm{~S}(2)$ & 2.0338 & $3.9 \pm 0.7$ & 2.4 \\
$\mathrm{H}_{2} 1-0 \mathrm{~S}(1)$ & 2.1218 & $9.4 \pm 0.2$ & 2.5 \\
$\mathrm{Br} \gamma$ & 2.1661 & $14.3 \pm 3.5$ & 11.6 \\
$\mathrm{Br} \gamma$ (narrow) & 2.1661 & $4.8 \pm 1$ & - \\
$\mathrm{H}_{2} 1-0 \mathrm{~S}(0)$ & 2.2233 & $2.4 \pm 1.2$ & - \\
\hline
\end{tabular}

Notes. ${ }^{(a)}$ Wavelengths are given in the rest frame. ${ }^{(b)}$ This paper. (c) From Gilli et al. (2000), aperture size $1^{\prime \prime} \times 2^{\prime \prime}$.

morphological large scale major $\left(\mathrm{PA}=34.62^{\circ}\right)$ and minor axes $\left(\mathrm{PA}=-55.38^{\circ}\right)$, respectively. The exposure time amounts to 40 min per slit position. The spectra were reduced using standard techniques carried out by the ESO pipeline software.

In order to determine line positions, and subsequently radial velocities of $\mathrm{H}_{2} 1-0 \mathrm{~S}(1)$, a spectrum from the outer edge of the galaxy's major or minor axes was extracted, the signal-to-noise determined and if necessary the spectrum from the adjoining pixel added until a $S / N=3$ was reached. This procedure was repeated along the slit to the opposite edge of the galaxy resulting in a set of 114 and 62 individual spectra along the major and minor axes, respectively. $\operatorname{Br} \gamma(2.16 \mu \mathrm{m})$ can also be detected in these spectra. These 2D-data sets were then analysed in a similar way to their 3D counterparts (see Sect. 5).

\section{Observational results}

\subsection{The spectrum of NGC 2992}

In Fig. 1 we show the SINFONI spectrum of NGC 2992 integrated over the whole FOV of $3^{\prime \prime} \times 3^{\prime \prime}$ centered on the nucleus. The most prominent lines are $\mathrm{H}_{2} 1-0 \mathrm{~S}(3)$ at $1.958 \mu \mathrm{m}$, [Si VI] at $1.96 \mu \mathrm{m}, \mathrm{H}_{2} 1-0 \mathrm{~S}(1)$ at $2.12 \mu \mathrm{m}$, and $\mathrm{Br} \gamma$ at $2.16 \mu \mathrm{m}$, other molecular hydrogen transitions and some $\mathrm{CO}$ bandheads are also present. The line fluxes are summarized in Table 1 together with line fluxes from Gilli et al. (2000).

Variability of the infrared flux of NGC 2992 was already reported by Glass (1997). Apart from an outburst in 1988, which 

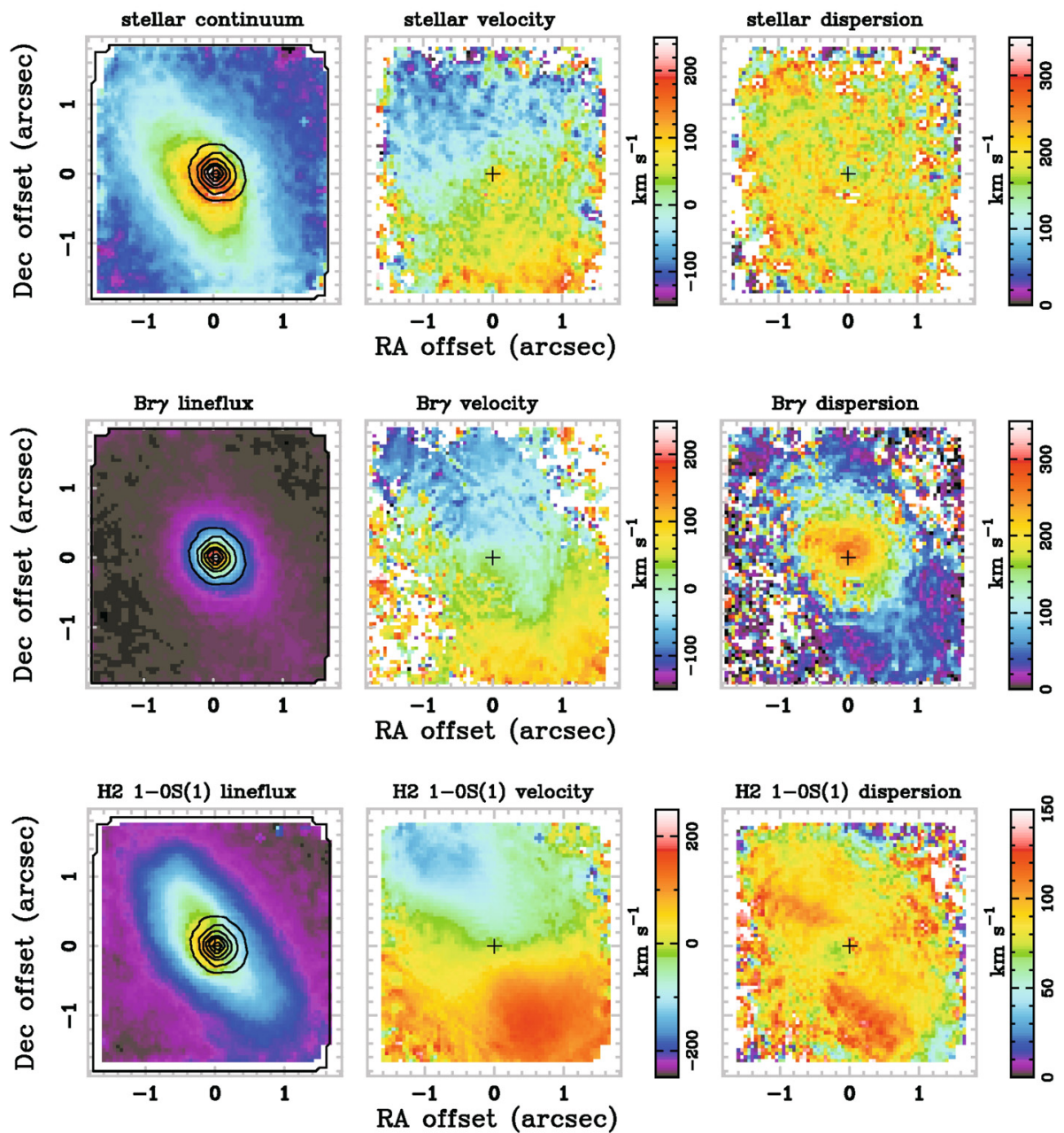

Fig. 2. Continuum, velocity, and dispersion maps of NGC 2992 (left to right) of stars, $\mathrm{Br} \gamma$, and $\mathrm{H}_{2} 1-0 \mathrm{~S}(1)$ (top to bottom). 1" corresponds to $150 \mathrm{pc}$. The contour lines of the $\mathrm{Br} \gamma$ continuum are overlayed on all continuum maps and a cross indicates the center of each map. North is up and east to the left.

was especially strong in the $K$ - and $L$-band, he reported a fading of NGC 2992 by about $20 \%$ in the $K$-band, $50 \%$ in the $L$-band between 1978 and 1996, and by a factor of $\approx 20$ in X-rays at the same time. Then, in 1998 BeppoSAX observations showed the X-ray flux to be again similar to that of 1978 (Gilli et al. 2000). Also line properties of Balmer- and Paschen-lines in the near infrared were similar to those observed before the decline. It is obvious from Table 1 that our line fluxes from 2005 (determined for a similar aperture size) are higher than those of Gilli et al. (2000) from January 1999. Unfortunately we have no information about $\mathrm{H} \alpha$ and $\mathrm{Pa} \beta$ to compare them to former observations given in Gilli et al.

Oliva et al. (1995) show that the ${ }^{12} \mathrm{CO}(2-0)$ at $2.2 \mu \mathrm{m}$ in the $K$-band, and the ${ }^{12} \mathrm{CO}(6-3)$ bandhead at $1.6 \mu \mathrm{m}$ in the $H$-band, can be used to separate stellar and non-stellar continua in AGN. However, doing so requires high signal-to-noise data in both bands. Instead, we follow the method of Davies et al. (2007) which uses just the ${ }^{12} \mathrm{CO}(2-0)$ bandhead to calculate the fraction of the nuclear flux that is stellar. By assuming that the stellar equivalent width in the nuclear region is about $12 \AA$, regardless of the star formation history, as predicted by the models created with the stellar synthesis code STARS (Sternberg 1998; Sternberg et al. 2003), which is comparable to Starburst99 (Leitherer et al. 1999; Vazquez \& Leitherer 2005), we calculated the non-stellar dilution using the equation:

$1-D=\frac{E W_{\text {obs }}}{E W_{\text {intr }}}$ where $D$ is the fraction of the continuum that is non-stellar. We found an equivalent width of $(5.0 \pm 1.6) \AA$ in the central $0.5 \times 0.5$, implying that $58 \%$ of the $K$-band luminosity in this region comes from non-stellar components. With this value we determined a stellar luminosity of $L_{K}=3.9 \times 10^{7} L_{\odot}$ and a total continuum luminosity of $L_{K}^{\text {cont }}=5.4 \times 10^{7} L_{\odot}$ in this central region in the $K$-band.

\subsection{2-dimensional flux distributions, velocities, and velocity dispersions}

2-dimensional flux distributions, velocities, and velocity dispersions of individual lines were determined by fitting the unresolved line profile of an $\mathrm{OH}$ line, which was convolved with a Gaussian, to the respective lines. The continuum level was found by a linear fit to the spectrum and subtracted. The uncertainty of a fit was estimated using $\chi^{2}$ techniques by refitting the best-fit Gaussian with added noise at the same level as the data 100 times. Standard deviations of the best-fit parameters are then used as the uncertainties. Uncertainties of velocities and velocity dispersions are of the order of $10 \mathrm{~km} \mathrm{~s}^{-1}$ to $15 \mathrm{~km} \mathrm{~s}^{-1}$ for the ${ }^{12} \mathrm{CO}(2-0)$ bandhead at $2.2 \mu \mathrm{m}$ (i.e. for the stars) and $\mathrm{Br} \gamma$, and about $5 \mathrm{~km} \mathrm{~s}^{-1}$ for $\mathrm{H}_{2} 1-0 \mathrm{~S}(1)$. Errors of the continuum range from below $1 \%$ for $\mathrm{Br} \gamma$ and $\mathrm{H}_{2} 1-0 \mathrm{~S}(1)$, and $6 \%$ for the stars.

In Fig. 2 we show the morphology of the stellar continuum, the $\mathrm{Br} \gamma-$ and $\mathrm{H}_{2} 1-0 \mathrm{~S}(1)$ lines and their respective velocities and velocity dispersions. The spatial resolution is $0.3^{\prime \prime}$ corresponding to a length scale of about $50 \mathrm{pc}$; the spectral resolution is 

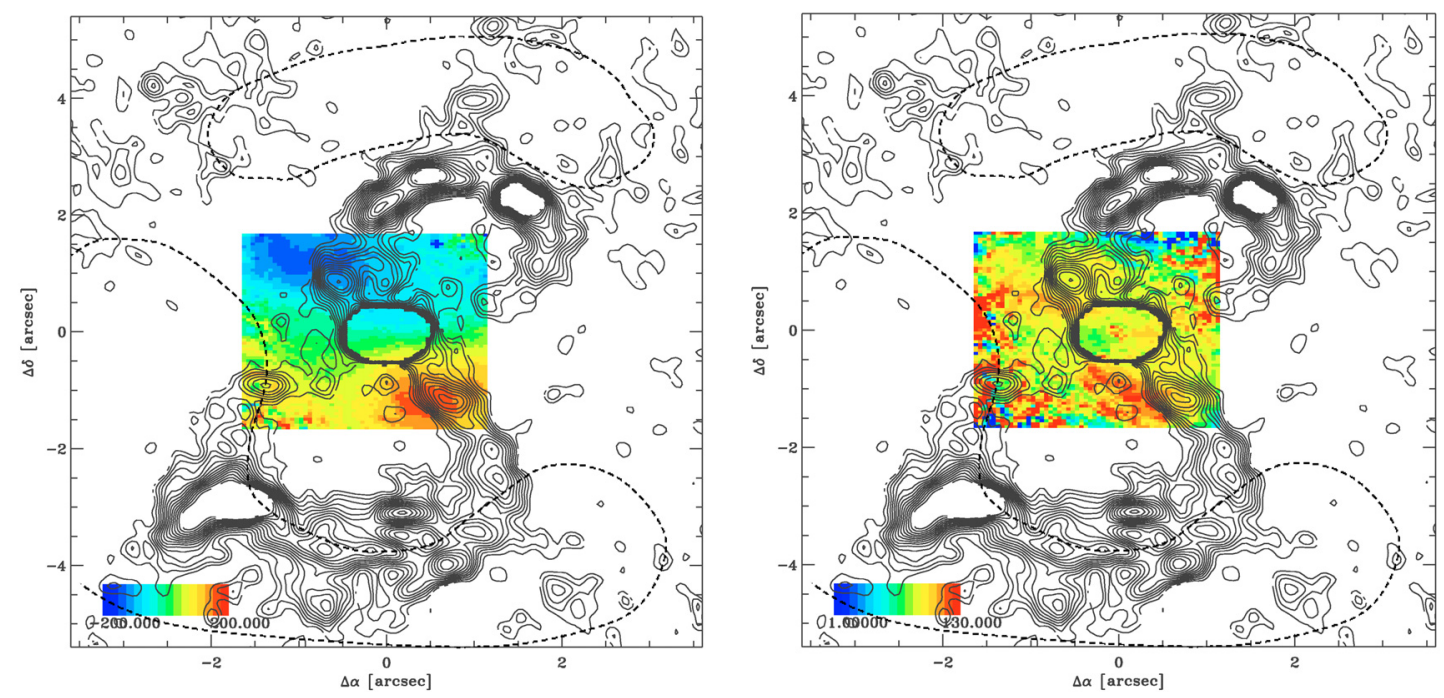

Fig. 3. Velocity (left) and velocity dispersion maps (right) of $\mathrm{H}_{2} 1-0 \mathrm{~S}(1)$ with the contours of the radio flux at $8.46 \mathrm{GHz}$ (Leipski et al. 2006, courtesy Heino Falcke) and the rough positions of the redshifted and blueshifted velocity residuals of [O III] (dashed lines at bottom and top) found by García-Lorenzo et al. (2001) overlayed. North is up and east to the left.

$83 \mathrm{~km} \mathrm{~s}^{-1}$. The continuum seems to trace an inclined disk with the north-west side more obscured. Given the broad dust lane seen in optical images this is not surprising.

There exist remarkable differences. Firstly, the morphology of the $\mathrm{Br} \gamma$ line does not follow the stellar continuum. To make this more obvious the contour lines of the $\mathrm{Br} \gamma$ continuum are overlaid in Fig. 2 on the stellar continuum. The center of the stellar continuum is shifted to the south east and the intensity distribution is elongated in the north east to south west direction in contrast to the circular Br $\gamma$ contour. This means that part of the $\mathrm{Br} \gamma$ emission must be attributed to other sources than a starburst. On the other side, the morphology of the $\mathrm{H}_{2} 1-0 \mathrm{~S}(1)$ line is similar to the stellar continuum.

The zero line of the velocity is orientated roughly east west in all three velocity maps. The maximum velocities range from $+100 \mathrm{~km} \mathrm{~s}^{-1}$ to $+170 \mathrm{~km} \mathrm{~s}^{-1}$ and the minimum velocities from $-100 \mathrm{~km} \mathrm{~s}^{-1}$ to $-140 \mathrm{~km} \mathrm{~s}^{-1}$. The velocity map of the stars shows a distinct increase to up to $100 \mathrm{~km} \mathrm{~s}^{-1}$ at the position of the centre of the $\operatorname{Br} \gamma$ continuum.

The stellar component, $\mathrm{Br} \gamma$ and $\mathrm{H}_{2}$ 1-OS(1) all show velocities away from us in the southwest and towards us in the northeast. The velocity field of $\mathrm{H}_{2} 1-0 \mathrm{~S}(1)$ and also that of $\mathrm{Br} \gamma$, albeit not as clearly, show redshifted emission also to the southeast. García-Lorenzo et al. (2001) investigated the velocity field of [O III] and found an arc of redshifted velocities to the southeast and one of blueshifted velocities to the northwest with velocities of $(64 \pm 18) \mathrm{km} \mathrm{s}^{-1}$, and $(-79 \pm 28) \mathrm{km} \mathrm{s}^{-1}$, respectively. These arcs are just outside our field of view. Only the redshifted velocities in the south east corner of $\mathrm{H}_{2}$ might be caused by them (Fig. 3). Since these "arcs" resemble the loops of the figure-of- 8 when superimposed on the radio emission there might be a relation between both structures. However, this would suggest an inflow, if the southeast part of the figure-of- 8 is closer to us, as generally assumed. In the picture of expanding gas bubbles this cannot be understood.

Already Chapman et al. (2000) proposed that the radio morphology consists of a component out of the plane of the galaxy disk and one within the disk associated with a spiral arm. On the basis of the narrow and well defined spectral lines within the arcs García-Lorenzo et al. conclude that the arcs are due to gas in the disk rather than in the loops. However, none of the arcs is associated with the structures that Chapman et al. interpret as spiral arms.

There is a small region in the velocity field of $\operatorname{Br} \gamma$ which extends from west of the centre to nearly the edge of the FOV in the SW with a lower velocity relative to its surroundings. This can be interpreted as an outflow superimposed on the galaxy rotation. Although it coincides with the southern inner spiral arm found by Chapman et al. (2000) in their model-subtracted $R$ and $H$-band images, it cannot be related to it because then the spiral arm would move relative to the ambient medium. There is no counterpart in the $\mathrm{H}_{2} 1-0 \mathrm{~S}(1)$ and stellar continuum velocity fields.

Common to both the stars and the lines are relatively high velocity dispersions of up to $250 \mathrm{~km} \mathrm{~s}^{-1}$ for the stars and $\mathrm{Br} \gamma$, and $130 \mathrm{~km} \mathrm{~s}^{-1}$ for $\mathrm{H}_{2} 1-0 \mathrm{~S}(1)$. However, the stellar dispersion map shows no structures, except that there seems to be a local minimum at the position of the centre of the $\mathrm{Br} \gamma$ continumm. There, the measured velocities range from $(110-150) \mathrm{km} \mathrm{s}^{-1} \pm$ (20-30) $\mathrm{km} \mathrm{s}^{-1}$ compared to the surrounding values of more than $(200 \pm 15) \mathrm{km} \mathrm{s}^{-1}$. This high stellar velocity dispersion which we observe even on the smallest scales suggests that the $K$-band light is dominated by stars in the bulge.

The high velocity dispersion of $\mathrm{Br} \gamma$ is confined to the inner $1^{\prime \prime} 2$, outside dropping to $(0-30) \mathrm{km} \mathrm{s}^{-1}$. The dispersion of $\mathrm{H}_{2} 1-0 \mathrm{~S}(1)$ shows maxima of $150 \mathrm{~km} \mathrm{~s}^{-1}$ to the southwest and northeast of the centre, which correspond with the velocity maxima and minima. They also lie at the edge of radio features within the loops (Fig. 3) and may be associated with inner spiral arms. The dispersion of $\mathrm{H}_{2} 1-0 \mathrm{~S}(1)$ also shows a local minimum at the centre where the dispersion drops to $70 \mathrm{~km} \mathrm{~s}^{-1}$. There might also be a local maximum at the position of the centre of the $\mathrm{Br} \gamma$ contour.

The complexity and the differences of velocity and dispersion maps of the stars and the lines might be due to the superposition of the galaxy rotation and an outflow as it was already suggested by several authors (e.g. Chapman et al. 2000).

\section{Constraining nuclear star formation in NGC 2992}

The possible existence of a nuclear starburst in NGC 2992 was investigated by Davies et al. (2007), who compared the radial 

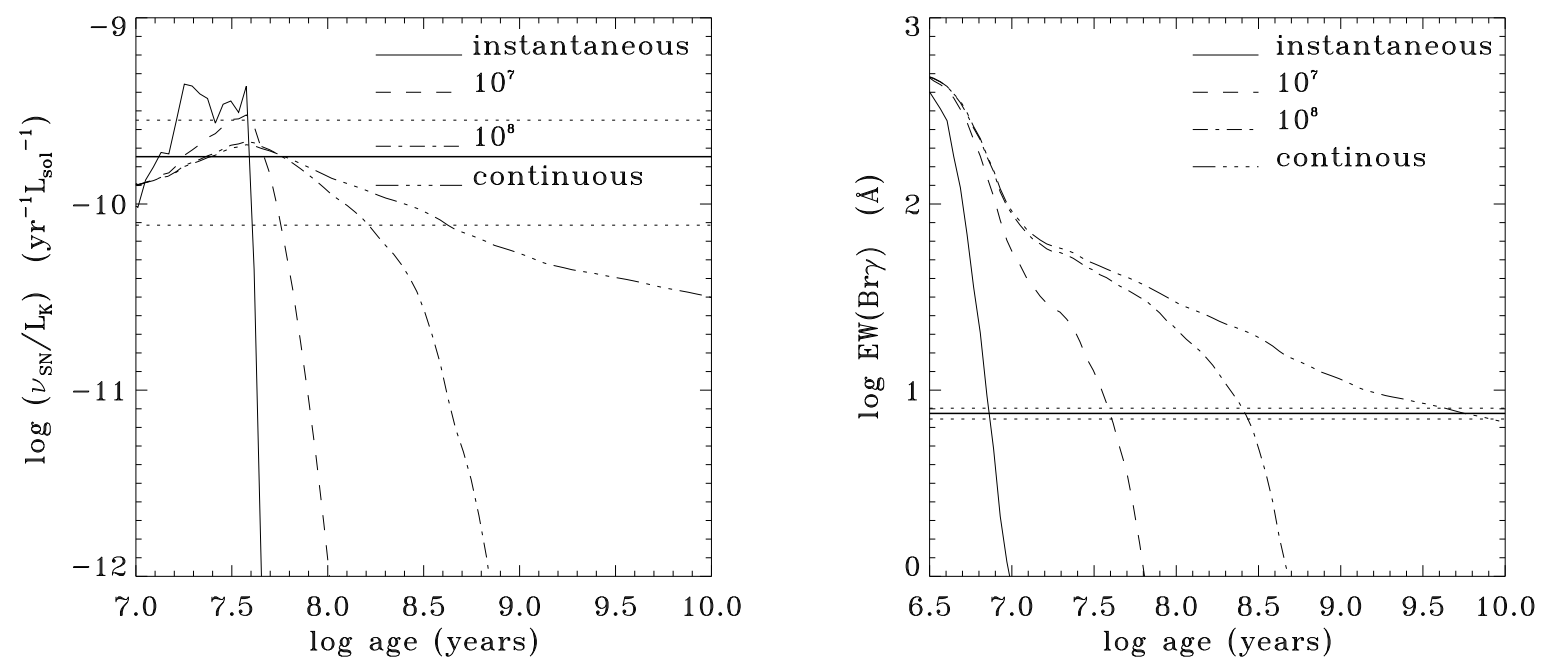

Fig. 4. Supernova rate and $\operatorname{Br} \gamma$ equivalent width calculated with STARS as a function of age for several star formation timescales. They are normalised to the $K$-band stellar continuum and $L_{K}$ is the total luminosity in the $1.9-2.5 \mu \mathrm{m}$ band in units of the solar luminosity $\left(L_{\mathrm{bol}}=3.8 \times\right.$ $10^{26} \mathrm{~W}$ ). The observed values are drawn as horizontal lines, the error margin is indicated by dashed lines.

profile of the stellar continuum to an $r^{1 / 4}$ law and an exponential profile. They found that there might be excess continuum at $r<0$ '.5 due to a distinct stellar population superimposed on the bulge. However, although the size scale matched the nuclear star forming regions in other AGN with typical $H W H M$ of $10-50 \mathrm{pc}$ (s. Fig. 5 in Davies et al. 2007), they did not reach a conclusion on NGC 2992 because the excess was only apparent for an exponential profile. Data at a higher resolution of about 0 .' 1 would be needed to explore the spatial properties further. Instead, here we look at various star formation diagnostics in order to assess the luminosity and age of a putative nuclear starburst.

The stellar population synthesis code STARS (e.g. Sternberg 1998; Sternberg et al. 2003) was also used to model the equivalent width of $\operatorname{Br} \gamma$ and the supernova rate. The program follows the evolution of a cluster of stars through the HertzsprungRussell-Diagram depending on the star formation history. We assumed decaying star formation rates with timescales of $10^{6}$ (instantaneous), $10^{7}, 10^{8}$, and $10^{12}$ (continuous) years. In Fig. 4 the results from STARS together with the observations are presented. Further details on star formation diagnostics can be found in Davies et al. (2007).

\section{1. $\mathrm{Br} \gamma$ equivalent width and supernova rate}

Using the stellar continuum luminosity from Sect. 3.1, an upper limit for the equivalent width of $\mathrm{Br} \gamma$ associated with star formation from the narrow $\mathrm{Br} \gamma$ line flux can be estimated. However, for NGC 2992 it is difficult to quantify what fraction of $\mathrm{Br} \gamma$ is associated with star formation, since the morphology of the line does not follow the stars (Fig. 2). Especially the south-west side shows velocities which are blue shifted relative to their environment indicative of motions towards us. The different morphology of $\mathrm{H}_{2} 1-0 \mathrm{~S}(1)$ and $\mathrm{Br} \gamma$ found in Sect. 3.2 points towards a kinematically different origin, and supports the conclusion that at least part of the Br $\gamma$ emission is due to the AGN and not to star formation. Thus the narrow $\mathrm{Br} \gamma$ line flux includes a certain unknown fraction from the AGN and the derived equivalent width of $(7.5 \pm 0.5) \AA$ is only an upper limit. This value, and all the numbers throughout the rest of the paper, are determined in the central $0.5 \times 0$.' 5 unless otherwise specified.

We estimate a supernova rate for NGC 2992 from the unresolved radio flux of $7 \mathrm{mJy}$ at $5 \mathrm{GHz}$ (Wehrle \& Morris 1988) and the estimation done by Sadler et al. (1995) that the core flux at $5 \mathrm{GHz}$ is less than $6 \mathrm{mJy}$. Their estimation is based on their higher spatial resolution measurements at $2.3 \mathrm{GHz}$ and the nondetections at $1.7 \mathrm{GHz}$ and $8.4 \mathrm{GHz}$. Taking a flat spectral index (Chapman et al. 2000) the $5 \mathrm{GHz}$ flux will not be much less than $6 \mathrm{mJy}$, which would result in about $1 \mathrm{mJy}$ extended emission. If we assume that it can be attributed to star formation, a supernova rate of $0.003 \mathrm{yr}^{-1}$ can be derived with the relation of Condon (1992). On the other hand, if we assume a spectral index of 0.82 , which was derived by Ulvestad \& Wilson (1984) for the unresolved flux at $1.5 \mathrm{GHz}$ and $5 \mathrm{GHz}$, together with the $2.3 \mathrm{GHz}$ flux of Sadler et al. (1995) we get a flux of about $3 \mathrm{mJy}$ at $5 \mathrm{GHz}$. This leaves us with about $4 \mathrm{mJy}$ extended emission corresponding to a supernova rate of $0.011 \mathrm{yr}^{-1}$. We consider the derived supernova rates as extremes and adopt a mean value of $0.007 \mathrm{yr}^{-1}$.

We can now try to determine a star formation age from the observed $\operatorname{Br} \gamma$ equivalent width and the supernova rate by comparing them to the theoretical values calculated by STARS, which are plotted in Fig. 4 for different star formation timescales. It is immediately obvious that for a continous star formation the observed $\mathrm{Br} \gamma$ equivalent width and the supernova rate yield inconsistent star formation ages of more than $3 \mathrm{Gyr}$ and (25-50) Myr, respectively. In addition, an ongoing star formation would require an equivalent width of $\operatorname{Br} \gamma$ of $10-15 \AA$, which is not observed.

An intermediate decay timescale of $10^{8} \mathrm{yr}$ yields a star formation age from the $\mathrm{Br} \gamma$ equivalent width of about $280 \mathrm{Myr}$ and 25-60 Myr from the supernova rate, which is an order of magnitude lower. Even if we consider the lower boundary of the supernova rate of $0.003 \mathrm{yr}^{-1}$ the resulting star formation age of $160 \mathrm{Myr}$ is well below the age derived from the $\operatorname{Br} \gamma$ equivalent width.

The best agreement between the derived star formation ages from the $\mathrm{Br} \gamma$ equivalent width and the supernova rate can be achieved for a decay timescale of $10^{7} \mathrm{yr}$. The resulting star formation ages amount to $40 \mathrm{Myr}$ and $50 \mathrm{Myr}$ respectively. The largest error of $\pm 10 \mathrm{Myr}$ is due to the uncertain radio flux at $5 \mathrm{GHz}$ and subsequently the supernova rate. The unknown contribution of the AGN to Br $\gamma$ does not contribute significantly to the uncertainty, because the curve is steep. Even an increasing contribution of the AGN up to nearly $100 \%$, which leads to a 
respective lower stellar contribution to the $\mathrm{Br} \gamma$ equivalent width, would increase the age only up to $60 \mathrm{Myr}$. This is still within the errors set by the supernova rate. We therefore conclude that a stellar population exists in NGC 2992 with an age of $40 \mathrm{Myr}$ to 50 Myr.

In principal there is also agreement between the $\mathrm{Br} \gamma$ equivalent width and the supernova rate for instantaneous star formation at a very young age of about $10 \mathrm{Myr}$, but we consider this less likely; partly because the timescales are much shorter, but primarily because it requires very careful tuning to match the increasing supernova rate and the quickly decaying $\operatorname{Br} \gamma$ equivalent width.

Combining the age range of $40-50 \mathrm{Myr}$ with the bolometric luminosity and black hole mass for NGC 2992 allows us to locate this galaxy in Fig. 11 of Davies et al. (2007). This figure shows how the luminosity of an AGN might be related to the age of the starburst, and NGC 2992 can be placed in the lower left where the starburst age is young and the AGN is, at the present time, accreting at a rate that is low compared to the Eddington rate. The galaxy thus appears to fit into the proposed scheme, where there is either a delay between the onset of starburst activity and the onset of AGN activity, or star formation ceased once the black hole become active (s. Davies et al. 2007, for a detailed discussion).

We also briefly consider whether the interaction between NGC 2992 and NGC 2993 might be responsible for the starburst. The gravitational (tidal) force during an interaction can be approximated as a delta-function that occurs at perigalacticon. According to Duc et al. (2000) perigalacticon of NGC 2992 and NGC 2993 occurred about $100 \mathrm{Myr}$ ago. At that time the gas would have felt the strongest impulse. Given that it takes time for the gas to respond and, for example, be driven to the centre, it is perhaps plausible for the interaction to have triggered the starburst.

\subsection{Mass-to-light-ratio and bolometric luminosity}

In order to estimate the mass-to-light-ratio we determined the dynamical mass within 0.5 from the following relation:

$M_{\mathrm{dyn}}=\left(v_{\mathrm{rot}}^{2}+3 \sigma^{2}\right) \cdot R / G$

where $v_{\text {rot }}$ is the observed velocity corrected by the inclination and $\sigma$ is the observed velocity dispersion at radius $R$ (Bender et al. 1992; Davies et al. 2007). We did not correct for the molecular gass mass nor for the contribution of an old stellar population. Therefore the derived dynamical mass of $8 \times 10^{7} M_{\odot}$ is only an upper limit. With a black hole mass of $5 \times 10^{7} M_{\odot}$ determined from velocity dispersion measurements (Woo \& Urry 2002) the dynamical mass is at most $3 \times 10^{7} M_{\odot}$ for the young population. Taken at face value, the resulting mass-to-light-ratio of 0.8 speaks in favour of a star formation age greater than $80 \mathrm{Myr}$ for all star formation rates. This is a factor of 2 higher than the age derived from the $\operatorname{Br} \gamma$ equivalent width and the supernova rate. However, it needs to be borne in mind that the dynamical mass is only an upper limit of the younger stellar population (it will contain a significant contribution from the older bulge population) and the uncertainty of the black hole mass can be nearly as high as 50\% (s. Woo \& Urry 2002). Either a change of the black hole mass by $10 \%$ or a reduction of the mass of the young population by $20 \%$ would bring the star formation ages into agreement.

STARS also allows us to estimate the stellar bolometric luminosity. The ratio between $L_{\mathrm{bol}}$ and $L_{\mathrm{K}}$ depends on the age and the exponential decay timescale of the star formation. Given the star formation age of 40-50 Myr we derive a bolometric luminosity for NGC 2992 of 70-100 times the $K$-band luminosity or $(3-4) \times 10^{9} L_{\odot}$, which is $(6-8) \%$ of the total bolometric luminosity of NGC $2992\left(5 \times 10^{10} L_{\odot}\right.$ calculated between 8 and $1000 \mu \mathrm{m}$ from IRAS $12-100 \mu \mathrm{m}$ flux densities, and additionally taking into account the emission at shorter wavelengths; Davies et al. 2007).

\subsection{Star formation rate}

Using the star formation history (decay timescale and age) above, we can estimate the star formation rate. The first step is to determine what fraction of the $K$-band luminosity in the central 0.5 comes from young stars. To do so, we estimate the contribution from an old population, by assuming that the dynamical mass is dominated by this old population. For an adopted age of 5 Gyr, STARS yields a mass-to-light ratio of about $30 M_{\odot} / L_{K}$. The dynamical mass determined above of $3 \times 10^{7} M_{\odot}$ then results in a luminosity of $1 \times 10^{6} L_{\odot}$ for the old population, one order of magnitude below the observed $K$-band luminosity. Therefore the $K$-band luminosity is dominated by the young population. We can therefore scale the luminosity of the starburst model to the total $K$-band luminosity in the central $0 . ' 5$, yielding an initial star formation rate of $4.3 \mathrm{M}_{\odot} \mathrm{yr}^{-1}$ for an age of $50 \mathrm{Myr}$. Since the SFR is decaying, an alternative, perhaps more meaningful, number may be the time-averaged star formation rate - which we define as simply the mass of stars formed divided by the age. This yields $\langle S F R\rangle \sim 1 M_{\odot} \mathrm{yr}^{-1}$. While this appears modest, in terms of SFR per unit area it corresponds to $\sim 200 M_{\odot} \mathrm{yr}^{-1} \mathrm{kpc}^{-2}$, within the range of values found for the nuclear starbursts in other nearby AGN by Davies et al. (2007).

An independent method to estimate the SFR is via the polycyclic aromatic hydrocarbon (PAH) features. Farrah et al. (2007) employed diagnostics based on the luminosities and equivalent widths of fine-structure emission lines and PAH features as well as the strength of the $9.7 \mu \mathrm{m}$ silicate absorption feature to investigate the power source of the infrared emission in ULIRGs. With these tools they were able to distinguish between starburst and AGN caused infrared emission, and to determine a star formation rate.

In order to apply these diagnostics to NGC 2992 we used near infrared pipeline reduced IRS spectra from the Spitzer archive. If more than one spectrum was available we took the mean value from all spectra to determine line luminosities. $25 \mu \mathrm{m}$ and $60 \mu \mathrm{m}$ fluxes were taken from the IRAS Revised Bright Galaxy Sample (Surace et al. 2004). The results are given in Table 2.

The star formation rate

$$
\operatorname{SFR}\left[M \cdot \mathrm{yr}^{-1}\right]=1.18 \times 10^{-41}\left(L_{6.2 \mu \mathrm{m}}+L_{11.2 \mu \mathrm{m}}\right)\left[\mathrm{erg} \mathrm{s}^{-1}\right]
$$

was empirically derived from the PAH $6.2 \mu \mathrm{m}+11.2 \mu \mathrm{m}$ luminosity for a star formation occurring on timescales of $10^{7}-10^{8}$ years (Farah et al. 2007). It yields $2.5 M_{\odot}$ per year or about $10 M_{\odot} \mathrm{yr}^{-1} \mathrm{kpc}^{-2}$, if we take the slit width of 3.' 6 of the IRS spectra as the size of the investigated region for NGC 2992. This value is consistent with the SFR derived above, being above the time averaged rate but below the initial peak rate. While it can be regarded as fairly normal compared to the star formation rates of $100 M_{\odot}$ per year for nearby luminous starbursts (e.g. Weedman \& Houck 2008), in terms of SFR per unit area, it is as extreme as these luminous starbursts. 
Table 2. Measured line luminosities of fine-structure lines and PAHs for NGC 2992.

\begin{tabular}{lrr}
\hline \hline Line & $\begin{array}{r}\lambda^{a} \\
(\mu \mathrm{m})\end{array}$ & $\begin{array}{r}\text { luminosity } \\
\left(10^{33} \mathrm{~W}\right)\end{array}$ \\
\hline PAH & 6.2 & $9.2 \pm 0.7$ \\
PAH & 11.2 & $12.5 \pm 0.2$ \\
{$[\mathrm{~S} \mathrm{IV}]$} & 10.5 & $2.0 \pm 0.01$ \\
{$\left[\mathrm{~S} \mathrm{III}^{\mathrm{I}}\right]$} & 18.7 & $3.8 \pm 0.3$ \\
{$[\mathrm{Ne}$ II $]$} & 12.8 & $6.8 \pm 0.9$ \\
{$[\mathrm{Ne} \mathrm{III}]$} & 15.6 & $7.1 \pm 0.4$ \\
{$[\mathrm{Ne} \mathrm{V}]$} & 14.3 & $3.9 \pm 1.1$ \\
{$[\mathrm{O}$ IV $]$} & 25.9 & $14.7 \pm 1.6$ \\
\hline
\end{tabular}

Notes. ${ }^{(a)}$ Wavelengths are in the rest frame.

\subsection{Starformation versus AGN contribution}

The strength of the $7.7 \mu \mathrm{m}$ PAH feature $(1.7 \pm 0.02)$ indicates that the AGN in NGC 2992 contributes more than $50 \%$ to the infrared flux between $1 \mu \mathrm{m}$ and $1000 \mu \mathrm{m}$ (Genzel et al. 1998). [Ne V] at $14.32 \mu \mathrm{m}$, which is strong in AGN spectra but not in star forming regions, and [O IV], which is strong in star forming regions, are both present in the spectra of NGC 2992, pointing to a co-existing starburst and AGN activity.

In order to quantify this a little more we followed Farrah et al. (2007) and compared the equivalent width of the PAH at $6.2 \mu \mathrm{m}(E W=(0.084 \pm 0.002) \mu \mathrm{m})$ to the ratio of [Ne V] to [Ne II] and [O IV] to [Ne II], respectively. From these diagnostics we derive a 10-20\% starburst fraction and about 50\% AGN fraction to the infrared flux for NGC 2992 in agreement with the result from the PAH $7.7 \mu \mathrm{m}$ feature. The AGN contribution can also be estimated from the ratio of the $25 \mu \mathrm{m}$ flux to the $60 \mu \mathrm{m}$ flux against the $[\mathrm{Ne} \mathrm{V}]$ to $[\mathrm{Ne}$ II] ratio. Again the AGN contribution amounts to about $50 \%$.

In this and the previous section we utilized different methods to estimate the contribution of stellar, non-stellar and starburst components to the $K$-band and infrared flux $(1-1000 \mu \mathrm{m})$. Independent of wavelength range and FOV we found a 50-60\% AGN contribution. From the CO bandhead at $2.2 \mu \mathrm{m}$ we found that $42 \%$ of the $K$-band flux in the central $0.5 \times 0 . .5$ can be attributed to stars and from the star formation history that these stars must be young. Also in the $3^{\prime \prime} \times 3^{\prime \prime}$ FOV the $K$-band light is dominated by stars. However, according to the high stellar velocity dispersion it is more likely that stars from the bulge dominate in this larger FOV. Finally, we find from PAH line diagnostics in the infrared a 10-20\% contribution to the infrared flux from a starburst. In addition to the AGN and starburst components, there still remains about $20-40 \%$, which must be attributed to a third component - most likely the disk and bulge of the galaxy. This means that while the young starbust is important in the central tens of parsecs, it is still only a small part of the integrated luminosity of the galaxy. Therefore we conclude that in NGC 2992 we see a dominating AGN activity with a relatively minor contribution from a starburst.

\section{Origin of nuclear outflow}

\subsection{Starburst or AGN driven outflow}

Heckman et al. (1990) provided evidence that galaxies with a central starburst have large scale mass outflows which are presumably driven by the kinetic energy supplied by supernovae and winds from massive stars. In order to examine whether the outflow in NGC 2992 can be caused by the starburst we calculated the radius $r$ in kpc of a bubble which is inflated by energy injected at a constant rate and expanding into a uniform medium with a density $n_{0}$ in $\mathrm{cm}^{-3}$ (Heckman et al. 1990). From

$\mathrm{d} E / \mathrm{d} t \approx 3 \times 10^{41} r_{\mathrm{kpc}}^{2} v_{100}^{3} n_{0} \operatorname{erg~s~s}^{-1}$

with the velocity (in $100 \mathrm{~km} \mathrm{~s}^{-1}$ ) $v_{100} \approx 2$ (Allen et al. 1999), $n_{0} \approx 3 \mathrm{~cm}^{-3}$ (Heckman et al. 1990), and $\mathrm{d} E / \mathrm{d} t$ equal to the mechanical luminosity from stellar winds and SNe taken from starburst99 for instantaneous $\left(10^{40} \mathrm{erg} \mathrm{s}^{-1}\right)$ star formation we get a radius of $40 \mathrm{pc}$. This radius is too small compared to the observed radii of about $600 \mathrm{pc}$ of the figure-of- 8 and $2 \mathrm{kpc}$ for the outflows found in $\mathrm{H} \alpha$ and [O III] (Allen et al. 1999). An energy injection of $3 \times 10^{43} \mathrm{erg} \mathrm{s}^{-1}$ would be required to explain the observed radius and velocity of the outflow in NGC 2992. This estimation is based on a symmetric geometry. If we instead assume a cone with an opening angle of $116^{\circ}$, as seen for the large scale outflow (Allen et al. 1999), the energy needed is a factor of 10 lower but still cannot be provided by the starburst.

The $2 \mathrm{kpc}$ extension of the large scale outflow and the velocity of $200 \mathrm{kms}^{-1}$ suggest that the outflow originated in an event at most $10 \mathrm{Myr}$ ago. This is inconsistent with our derived star formation age of (40-50) Myr but in agreement with the typical timescale of the active phase of a black hole in an AGN. We note that although it is also consistent with the age for instantaneous star formation, this scenario we regarded as rather unlikely since it requires fine-tuning (see Sect. 4.1).

Thompson et al. (2005) showed that optically thick pressure supported starburst disks have a characteristic flux of about $10^{13} L_{\odot} \mathrm{kpc}^{-2}$. Above this limit matter would be blown away. With the derived luminosity of $(3-4) \times 10^{9} L_{\odot}$ within the central 0.'5 we estimated the characteristic flux for NGC 2992 to be $(5-7) \times 10^{11} L_{\odot} \mathrm{kpc}^{-2}$ which is well below this limit. Only when the starburst was at its peak luminosity, which for our preferred model is nearly 10 times greater than the current luminosity, would the star formation approach - although remaining below - this threshold. The conclusion from both energy and timescale considerations, is that for the starburst to drive the outflows it would have to be more intense and more recent - both of which are ruled out by our analysis.

Instead, we consider whether the outflows in NGC 2992 can be driven by the AGN. In order to estimate the AGN luminosity of NGC 2992 we use the spectral energy distribution (SED) of NGC 1068 as a template. For this SED, the optical $(750 \mathrm{~nm})$ to $100 \mathrm{keV}$ luminosity is a factor 11.8 greater than that in the 20-100 keV range (Pier et al. 1994). For NGC 2992, the luminosity in this latter range is $(1-4) \times 10^{43} \mathrm{erg} \mathrm{s}^{-1}$ (Beckmann et al. 2007). Thus the total luminosity of NGC 2992 is about an order of magnitude greater, which yields about $10^{44} \mathrm{erg} \mathrm{s}^{-1}$. This is a factor of 3-30 times greater than the energy estimated above that is required to generate the outflow. We therefore conclude that it is the AGN, rather than the starburst, that has driven the outflow.

\subsection{Velocities along the major axis}

Longslit spectra were taken with ISAAC along the major axis (position angle $\mathrm{PA}=34^{\circ}$ ) at $2.1 \mu \mathrm{m}$. The resulting velocity curve of $\mathrm{H}_{2} 1-0 \mathrm{~S}(1)$ along the slit shows a steep increase to the southwest (decline to NE) up to a radius of 1".5 away from the centre followed by a sharp decline within about 0.5 and subsequently a shallower incline. To the northeast the velocity curve behaves similar. This is also observed in the SINFONI data (Fig. 5), and is in agreement with published data of Marquez et al. (1998) for a position angle of $30^{\circ}$ and Veilleux et al. (2001), too. 


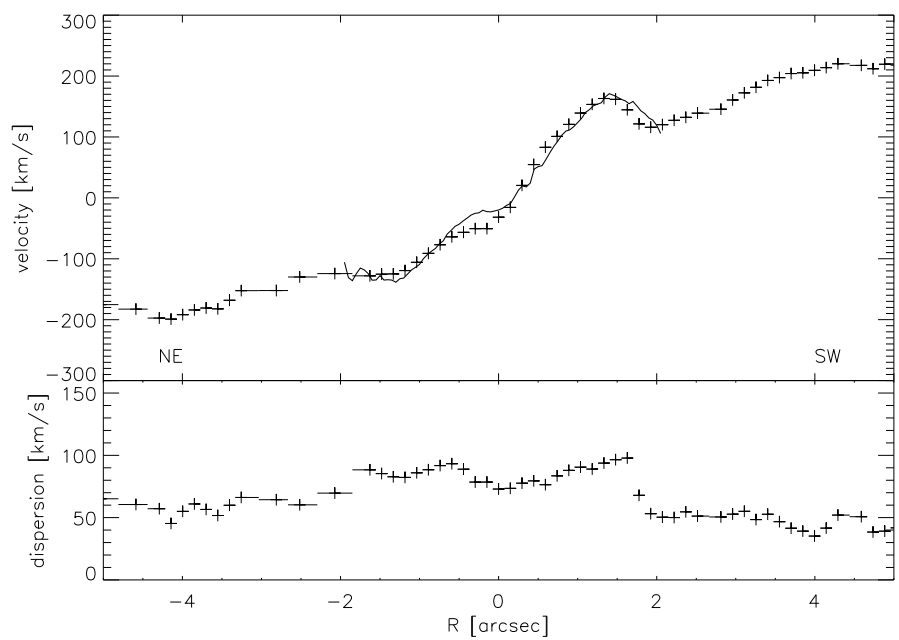

Fig. 5. Radial velocities (top) and corresponding velocity dispersion (bottom) from longslit spectra taken with ISAAC along the major axis of NGC 2992 (crosses). Overplotted is a radial velocity curve drawn from SINFONI data also along the major axis. $1^{\prime \prime}$ correspond to $150 \mathrm{pc}$, northeast is to the left, southwest to the right.

Colina et al. (1987) found asymmetric line profiles for a region with $r \leq 3^{\prime \prime}$, and symmetric profiles for $r \geq 3^{\prime \prime}$. They interpreted their results as dynamically decoupled nuclear and offnuclear regions. Their velocity curve of [O III] is in agreement with our velocity curve. However, due to their coarse sampling along the major axis they could not resolve less than $3^{\prime \prime}$.

Evidence for dynamical decoupled nuclear and off-nuclear regions comes also from kinemetry (Krajnovic̀ et al. 2006) of our 2-dimensional stellar velocity data. With the position angle and inclination as free parameters the PA changes from $45^{\circ} \pm 11^{\circ}$ to $22^{\circ} \pm 5^{\circ}$ at about $1^{\prime \prime}$ (Fig. 6). This is in good agreement with the $22.5^{\circ}$ of Jarrett et al. (2003) derived from the $K_{\mathrm{S}}$-band $20 \mathrm{mag} \mathrm{arcsec}^{-2}$ isophot. The PA of $45^{\circ}$ better fits to the inclination of the accretion disk of about $37^{\circ}$ and $46^{\circ}$, derived by Gilli et al. (2000) with the assumption of both Schwarzschild and Kerr metrics from disk line models and iron line profiles, than to the $34^{\circ}$ of García-Lorenzo et al. (2001). The PA of the 2-dimensional $\mathrm{H}_{2} 1-0 \mathrm{~S}(1)$ velocity data also changes at 1 ", however from $8^{\circ} \pm 4^{\circ}$ to $20^{\circ} \pm 4^{\circ}$ (Fig. 6). That the changes in PA are derived from kinematic rather than photometric data, argues that they are real and not just an effect of extinction. It therefore seems that the stars and the gas show a different behaviour in the very centre and a similar one outside.

\subsection{A geometric model for the inner $3^{\prime \prime} \times 3^{\prime \prime}$}

In order to investigate the different components contributing to the velocity field and to explain the different position angles observed in the central $3^{\prime \prime} \times 3^{\prime \prime}$ of NGC 2992 we developed a simple geometric model. It consists of an inner and an outer disk, in which particles were assumed to be in circular motion in a gravitational potential. The radius of the inner disk is determined by the change in PA found in kinemetry of stellar and $\mathrm{H}_{2} 1-0 \mathrm{~S}(1)$ data at about $1^{\prime \prime}$. It is not related to the starburst nor does it reflect the sphere of gravitational influence of the black hole, which is only a few parsec in radius. The radius of the outer disk is cut at $5^{\prime \prime}$ due to our FOV of $3^{\prime \prime}$. In addition, a conical outflow with constant velocity represents the figure-of- 8 . All components could be rotated by arbitrary angles around the $x$-, $y$-, and $z$-axis, which points to the observer, thus allowing for different orientations to each other. Velocities were adjusted in such a way, that they
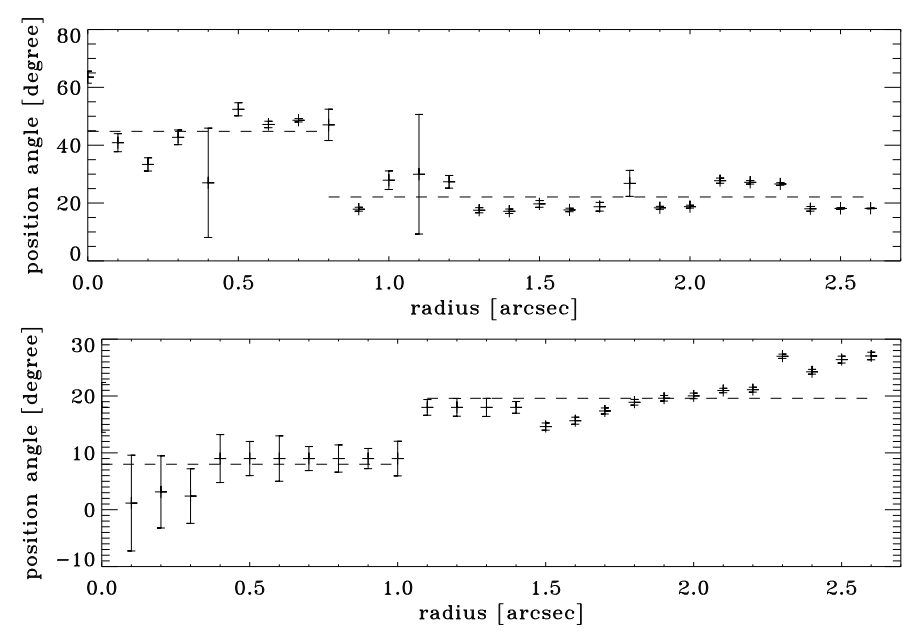

Fig. 6. The dependence of the position angle on the distance from the centre for the 2-dimensional stellar (top) and $\mathrm{H}_{2} 1-0 \mathrm{~S}(1)$ data (bottom). The mean values of the PA for radii below and above $1^{\prime \prime}$ are also given (dashed line).

reflect the observed velocity curve. Due to the simplicity of the model, we only can draw some qualitative conclusions.

There is an inner and outer region which must have different orientations in space (realized in the model by different rotation angles around the $x$-axis) in order to explain the different position angles. However, the inclination angle of $70^{\circ}$ and the position angles cannot be modelled exactly at the same time. Dependent on the start value for the ellipticity of the unrotated outer disk we get an inclination angle of $50^{\circ}$ for a circular disk and $63^{\circ}$ for an ellipticity of 0.7 , and position angles of $13^{\circ}$ and $18^{\circ}$, respectively. For the inner, circular region we get an inclination angle of $60^{\circ}$ and a position angle of $34^{\circ}$. The different inclination angles might point to a dynamical decoupled inner and outer region as it was already suggested by Colina et al. (1987).

In order to achieve a position angle of the figure-of- 8 consistent with the published $-26^{\circ}$ (Wherle et al. 1988), it has to be perpendicular to a plane whose inclination angle is $51^{\circ}$, which is slightly lower than the inclination of the inner disk. One can identify this plane with the plane of the accretion disk, which itself is not modeled. The resulting position angle is $-27^{\circ}$ with the SE part of it pointing away from us, consistent with the redshifted emission there seen by García-Lorenzo et al. (2001).

The derived geometry from our model yields the values from the literature of $15^{\circ}$ for the position angle of the outer region (photometric axis), $34^{\circ}$ for the inner $5^{\prime \prime}$ (García-Lorenzo et al. 2001), and the inclination of the accretion disk of $46^{\circ} \pm 7^{\circ}$ (Gilli et al. 2000) very well. The resulting velocity field from this geometry is shown in Fig. 8 and is in good agreement with the measured velocity field of $\mathrm{H}_{2} 1-0 \mathrm{~S}(1)$ depicted in the lower panel of Fig. 2. Within this geometry the hump in the velocity curve at 1 .'5 is the signature of the cone describing the figure-of- 8 , which is superposed on the inner disk. The stellar velocity curve along the major axis from SINFONI and ISAAC does not show these humps at 1 .'5 which speaks in favour of this interpretation. Our model suggests that the outer disk, inner disk, and accretion disk are at different orientations. One possible reason for this is that this geometry reflects a single warped disk, or even a warped outflow. However, it is beyond the scope of this paper to explore all the options and to assess the physical reason behind the observed geometry. 


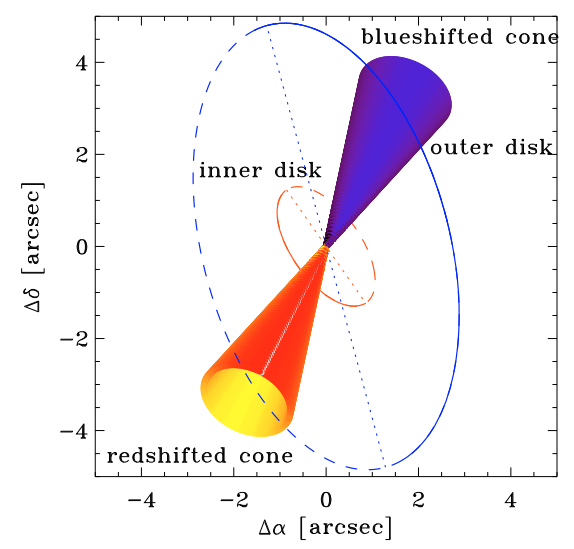

Fig. 7. Cartoon which shows the geometry of the central region of NGC 2992. Those parts of the disks with solid lines point towards us, those with long-dashed away from us. Short-dashed lines indicate the position angles of $15^{\circ}$ for the outer and $34^{\circ}$ for the inner disk.

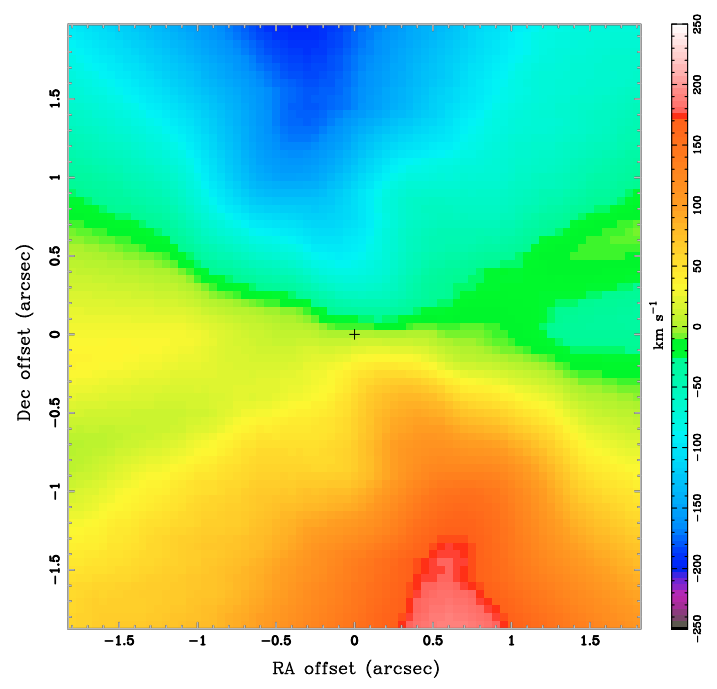

Fig. 8. Calculated velocity field in the central region of NGC 2992 resulting from two inclined disks and a conical outflow (see Sect. 5.3.).

\section{Conclusions}

We have presented a detailed analysis of near infrared $K$-band spectra complemented by $N$ - and $Q$-band spectra of NGC 2992. $K$-band spectra were obtained with the adaptive near infrared integral field spectrograph SINFONI and allow a reconstruction of the 2-dimensional distribution and kinematic of the stars and gas in the inner $3^{\prime \prime} \times 3^{\prime \prime}(450 \mathrm{pc})$ at an angular resolution of $0^{\prime} .3$. The $N$ - and $Q$-band data were obtained with Spitzer and taken from the archive. We compared the equivalent width of $\mathrm{Br} \gamma$ and the supernova rate, which was derived from radio data from the literature, to STARS evolutionary synthesis models and find evidence for a short burst of star formation (40-50) Myr ago.

From our near-infrared data as well as equivalent widths and luminosities of fine-structure emission lines and polycyclic aromatic hydrocarbon features detected in the $N$ - and $Q$-band spectra we were able to estimate the nuclear star formation rate and quantify the contribution of the star formation to the AGN luminosity: the luminosity is dominated by the AGN activity with a contribution of only 10-20\% from nuclear star formation. This is in agreement with the conclusion that part of the $\mathrm{Br} \gamma$ emission cannot be attributed to star formation but is due to the AGN.

Energy and timescale considerations let us conclude, that the starburst would have to be more recent and more intense to drive the figure-of- 8 as well as the large scale $(2 \mathrm{kpc})$ outflow. On the other hand the estimation of the luminosity of the AGN of $10^{44} \mathrm{erg} \mathrm{s}^{-1}$ is about an order of magnitude higher than is necessary to drive the outflows.

The observed velocity curve in $\mathrm{H}_{2}$ 1-0S(1) of NGC 2992 can be described as the superposition of the galaxy rotation and a conical outflow with constant velocity. The different position angles of the inner and outer region can be modelled by disks with different orientations in space and thus being probably dynamically decoupled. However, these disks are not related to the starburst. In this picture the south east cone is pointing away from us supporting the findings from García-Lorenzo (2001).

Acknowledgements. The authors thank all those at MPE and ESO Paranal who were involved in the SINFONI observations. This work is also based in part on observations made with the Spitzer Space Telescope, which is operated by the Jet Propulsion Laboratory, California Institute of Technology under a contract with NASA.

\section{References}

Abuter, R., Schreiber, J., Eisenhauer, F., et al. 2006, New A Rev., 50, 398

Allen, M. G., Dopita, M. A., Tsvetanov, Z. I., \& Sutherland, R. S. 1999, ApJ, 511,686

Beckmann, V., Barthelmy, S. D., Courvoisier, T. J.-L., et al. 2007, A\&A, 475, 827

Bender, R., Burstein, D., \& Faber, S. 1992, ApJ, 399, 462

Bonnet, H., Abuter, R., Baker, A., et al. 2004, The ESO Messanger, 117, 17

Chapman, S., Morris, S., Alonso-Herrero, A., \& Falcke, H. 2000, MNRAS, 314, 263

Colbert, E. J. M., Baum, S. A., O’Dea, C. P., \& Villeux, S. 1998, ApJ, 496, 786 Colina, L., Fricke, K. J., Kollatschny, W., \& Perryman, M. A. C. 1987, A\&A, 178,51

Condon, J. J. 1992, ARA\&A, 30, 575

Davies, R. I. 2007a, MNRAS, 375, 1099

Davies, R. I. 2007b, in The 2007 ESO Instrument Calibration Workshop, ESO Astrophys. Symp. [arXiv: astro-ph/0703044]

Davies, R. I., Mueller Sánchez, F., Genzel, R., et al. 2007, ApJ, 671, 1388

Duc, P.-A., Brinks, E., Springel, V., et al. 2000, AJ, 120, 1238

Eisenhauer, F., Abuter, R., Bickert, K., et al. 2003, in Instrument Design and Performance for Optical/Infrared Ground-based Telescopes, ed. M. Iye, \& A. Moorwood, Proc. SPIE, 4841, 1548

Farrah, D., Bernard-Salas, J., Spoon, H. W. W., et al. 2007, ApJ, 667, 149

García-Lorenzo, B., Arribas, S., \& Mediavilla, E. 2001, A\&A, 378, 787

Genzel, R., Lutz, D., Sturm, E., et al. 1998, ApJ, 498, 579

Gilli, R., Maiolino, R., Marconi, A., et al. 2000, A\&A, 355, 485

Glass, I. S. 1997, MNRAS, 292, L50

Heckman, T. M., Butcher, H. R., Miley, G. K., \& van Breugel, W. J. M. 1981, ApJ, 247, 403

Heckman, T. M., Armus, L., \& Miley, G. K. 1990, ApJS, 74, 833

Hicks, E. K. S., Davies, R. I., Malkan, M. A., et al. 2009, ApJ, 696, 448

Jarrett, T. H., Chester, T., Cutri, R., Schneider, S. E., \& Huchra, J. P. 2003, AJ, 125,525

Krajnovic̀, D., Cappellari, M., de Zeeuw, T., \& Copin, Y. 2006, MNRAS, 366, 787

Leipski, C., Falcke, H., Bennert, N., \& Hüttemeister, S. 2006, A\&A, 455, 161

Leitherer, C., Schaerer, D., Goldader, J. D., et al. 1999, ApJS, 123, 3

Márquez, I., Boisson, C., Durret, F., \& Petitjean, P. 1998, A\&A, 333, 459

Oliva, E., Origlia, L., Kotilainen, J. K., \& Moorwood, A. F. M. 1995, A\&A, 301, 55

Pier, E. A., Antonucci, R., Hurt, T., Kriss, G., \& Krolik, J. 1994, ApJ, 428, 124

Sadler, E., Slee, O., Reynolds, J., \& Roy, A. 1995, MNRAS, 276, 1273

Spoon, H. W. W., Marshall, J. A., Houck, J. R., et al. 2007, ApJ, 654, L49

Sternberg, A. 1998, ApJ, 506, 721

Sternberg, A., Hoffman, T., \& Pauldrach, A. 2003, ApJ, 599, 1333

Surace, J. A., Sanders, D. B., \& Mazzarella, J. M. 2004, AJ, 127, 3235

Thompson, T. A., Quataert, E., \& Murray, N. 2005, ApJ, 630, 167

Ulvestad, J. S., \& Wilson, A. S. 1984, ApJ, 285, 439

Vázquez, G. A., \& Leitherer, C. 2005, ApJ, 621, 695

Veilleux, S., Shopbell, P. L., \& Miller, S. T. 2001, AJ, 121, 198

Ward, M. J., Penston, M. V., Elvis, M., Maccacaro, T., \& Tritton, K. P. 1980, MNRAS, 193, 563

Weedman, D. W., \& Houck, J. R. 2008, ApJ, 686, 127

Wehrle, A. E., \& Morris, M., 1988, AJ, 95, 1689

Woo, J.-H., \& Urry, C. 2002, ApJ, 579, 530 